Current Research in Psychology 1 (1): 29-34, 2010

ISSN 1949-0178

(C) 2010 Science Publications

\title{
The Path to Treatment: Does It Matter? Relapse and the Reason for Seeking Treatment
}

\author{
Evelyn A. Cage and Annette S. Kluck \\ Auburn University 2084 Haley Center, Auburn, AL 36849
}

\begin{abstract}
Problem statement: Substance abuse is difficult to treat and many of those who struggle with substance abuse do not see treatment as necessary. Moreover, relapse is common among those who receive treatment. Family and friends of Individuals With Addictions (IWAs) sometimes use ultimatums to encourage the IWA to enter treatment. What is less clear is whether IWAs are more likely to relapse if they enter treatment due to an ultimatum as opposed to entering by their own choice. Exploring how effective IWAs perceive ultimatums to be in facilitating sustained sobriety based on their own personal experiences may help shed light on the utility of treatment obtained under these coerced conditions. Approach: IWAs were contacted through on-line support groups to participate in an internet based study exploring their substance abuse history and experiences with ultimatums. Eighty-one IWAs completed an on-line questionnaire designed to solicit quantitative and qualitative responses developed for the present descriptive study. Results: Three-fourths of participating IWAs who sought treatment due to an ultimatum subsequently relapsed and current length of sobriety was not related to sobriety attempts resulting from ultimatums. However, individuals who identified crack/cocaine as their substance of choice perceived ultimatums as more helpful than individuals who identified alcohol as their substance of choice. Emergent coding revealed that nearly half of participating IWAs reported that there were no benefits to ultimatums and $40 \%$ of those with personal experience with ultimatums reported no personal benefits. Conclusion/Recommendations: Overall, IWAs do not perceive ultimatums to be effective and they are likely to relapse when seeking treatment due to an ultimatum, though some IWAs believed ultimatums had benefits, suggesting that ultimatums may be more helpful depending on the particular characteristics of the IWA. Study findings suggested a need to identify other ways significant others can support IWAs in sobriety.
\end{abstract}

Key words: Intervention, ultimatum, addiction

\section{INTRODUCTION}

Substance dependence or addiction is a common and deadly illness that leaves many individuals in need of treatment interventions. Unfortunately, there are few illnesses as difficult to treat as addiction and for this reason, health care providers are at odds as to which treatment interventions are most efficacious in eliminating addictions, with many unproven treatment models still widely used (Campbell, 2007). To further complicate the treatment situation, many Individuals With Addictions (IWA's) do not welcome treatment as a necessary or viable option to address their problem. The perception that treatment is unnecessary among some IWA's has led to the development of treatment techniques such as motivational interviewing (Carroll et al., 2001). Such treatment involves the use of psychological principles in attitudinal change, particularly those grounded in cognitive and social psychology research. In other cases, individuals may simply refuse to enter treatment entirely. In instances such as these, family members often intervene to encourage the IWA to seek treatment. Out of feelings of desperation, this encouragement from the family is often given through the use of ultimatums. It is interesting to note that ultimatums are rarely used with clients that have other illnesses, highlighting the conflicting societal view of addiction as both an illness that needs treatment and the responsibility of the IWA (Hall, 1993).

The popular television series Intervention on the Arts and Entertainment (A and E) television network chronicles the use of ultimatums by families and friends of IWA's. At the show's climax, the IWA is led to believe they are attending a finale event. Instead, the IWA is coerced into a situation where they are confronted by their family and friends. Each family member/friend reads a statement, explaining what he/she will take away if the IWA does not attend

Corresponding Author: Evelyn A. Cage, Auburn University, 2084 Haley Center Auburn, AL 36849 
treatment. One way to think about these threats to take action is that the family members and friends are issuing ultimatums of what will happen if the IWA does not seek treatment. An ultimatum is defined as a final demand whose rejection will end negotiations and cause a resort to force or other direct action (MerriamWebster, 2003). After receiving ultimatums, nearly all participants on the show agree to treatment. What is less clear is how ultimatums may work without the televised setting or the long-term benefits of using this method to get a family member to enter treatment.

Although the accounts presented on the show Intervention are highly dramatized for television, using family members to support the entry of IWA's into treatment is fairly common. Models such as Community Reinforcement and Family Training (CRAFT) and the Pressure to Change Approach focus on instructing family members on how to best encourage IWA's to attend treatment by teaching family members behavioral procedures to interact with the IWA (Barber and Crisp, 1995; Copello et al., 2005; Meyers et al., 2005). Other techniques, such as The Johnson Intervention, have the family focus directly on the damage the addiction has caused and the ultimatums they will enact if treatment is not sought (Loneck et al., 1996). The Johnson Intervention is the intervention most closely associated with the television series Intervention and up to $80 \%$ of IWA's who undergo a Johnson Intervention enter treatment (Loneck et al., 1996). It is important to note that the primary goal of interventions such as CRAFT and The Johnson Intervention is to get the IWA into treatment (Meyers et al., 2005). Thus, there is an underlying assumption that the IWA can successfully obtain and sustain sobriety if treatment is sought. However, there is little known as to what conditions are necessary at the forefront if an IWA is to sustain sobriety after treatment. In other words, it is probable that certain conditions are necessary to increase the likelihood that an IWA will be successful in treatment (with success defined as both obtaining and sustaining sobriety) and it is probable that these conditions include aspects of the reason the IWA seeks treatment in the first place and the readiness of the IWA to change the addictive behavior (Laudet, 2003; Prochaska et al., 1992). It is clear that approaches similar to The Johnson Intervention are effective in getting an IWA into treatment. However, the empirical evidence does not support the use of ultimatums with regard to risk for relapse. In fact, many studies cite the opposite effect, such that relapse rates may be higher among those who enter treatment as the result of family behavior and family pressure may have no effect on motivation to change at all (Polcin and Beattie, 2007).
For instance, Matzger et al. (2005) found that interventions that include family interference can be negatively related to the length of sobriety for individuals suffering from alcoholism. Additionally, Loneck et al. (1996) found that IWA's that had undergone a Johnson Intervention were more likely to relapse than any other referral group of IWA's, including those that had been given ultimatums by a professional body such as a court or employer.

It is unclear why research seems to suggest that relapse is more common if family members give ultimatums. It may be due in part to the strained relationships that family interference/interventions can create between the IWA and their family members. Researchers have suggested that when family members treat alcoholics in an atypical fashion it intensifies feelings of isolation and leads to impoverished interpersonal relationships (Stead and Viders, 1979). In addition, Lavee and Altus (2001) found that men who were able to remain drug free in a 30 month period maintained closer relationships with their family members than those who relapsed within that 30 month period. This suggests that the supportive roles in familial relationships are critical to maintained sobriety (Marshall et al., 2005; Saatcioglu et al., 2006). However, it is not uncommon for relationships to be destroyed through the use of ultimatums.

This study will seek to address key issues in the dialogue concerning ultimatums and IWA's. First, is there greater risk for relapse if an IWA enters treatment due to ultimatums as opposed to entering treatment themselves? Furthermore, does this risk depend on some other characteristic associated with the IWA, such as drug of choice? We will explore these questions by assessing the perceptions IWA's have concerning ultimatums as tools to encourage treatment. Specifically, we will inquire about experiences with past ultimatums and the treatment outcomes associated with these experiences. It is our hope that this research will directly address the efficacy of using approaches such as the Johnson Intervention as well as offer insight as to characteristics of IWA's that might increase the likelihood of benefit or risk from such approaches.

\section{MATERIALS AND METHODS}

Participants: A total of 81 individuals who use online sobriety support group websites served as participants for this study. Some individuals indicated that their addiction did not involve alcohol/substance abuse (e.g., food addiction) and thus, they were not included in additional analyses. Our final sample consisted of 30 men and 47 women (77 total). 
Current Research in Psychology 1 (1): 29-34, 2010

Participants ranged in age from below 20-60-something with the majority being 30-59 $(\mathrm{n}=59)$. Most were Caucasian $(n=70 ; 90 / 9 \%)$. Three individuals identified as African American (3.9\%). Participants were predominantly from the Northeast $(\mathrm{n}=20 ; 26 \%)$ and Southeast $(\mathrm{n}=18 ; 23.4 \%)$ regions of the United States. An additional 26 were from other regions within the US and 13 individuals were from a country outside the US.

Procedure and measure: After obtaining IRB approval, an invitation to participate in the study was posted to on-online community websites, directing voluntary participants to an on-line questionnaire hosted by www.psychdata.com. Typically, posting the message required approval from the individual who hosted the support group website. Some individuals indicated they were not willing to post the invitation to the study (e.g., one was only willing to post information from members of AA). Individuals were provided information on the purpose of the study and indicated their consent by providing anonymous responses to a 20 item questionnaire developed for the present study. Questions required respondents to provide information about their background, attempts at sobriety and experiences with ultimatums. Perceptions of the usefulness of ultimatums were explored through quantitative and qualitative responses.

\section{RESULTS}

Descriptive statistics: Prior to conducting our analyses, we obtained descriptive information on drug use and sobriety experiences of our respondents. When asked to indicate their drug of choice, respondents most often reported preferring alcohol (Table 1) and the sample ranged from individuals at the start of their current sobriety as well as individuals with over 10 years being sober (Table 1). Over half of respondents (49) have seen the show Intervention, which depicts family and friends giving an addict an ultimatum. A total of 33 individuals have previously sought treatment or support (such as attending AA meetings) as a result of an ultimatum from a friend or family member while 30 individuals actually decided to get sober as the result of an ultimatum from friends/family. Over half (25) of those individuals who obtained sobriety due to an ultimatum from friends/family subsequently relapsed. Sixteen individuals described their current sobriety as the result of an ultimatum from friends/family. Table 1 also contains information about number of attempts at sobriety and number of times an individual got sober due to an ultimatum from a friend or family member.
Table 1: Substance abuse history frequencies of sample

\begin{tabular}{lrr}
\hline & No. of individuals & Respondents (\%) \\
\hline Drug of choice & 50 & \\
Alcohol & 10 & 64.9 \\
Crack/cocaine & 7 & 13.0 \\
Heroin/opiates & 7 & 9.1 \\
Polysubstance use & 3 & 9.1 \\
Marijuana & & 3.9 \\
Length of sobriety & 8 & \\
<1 week & 5 & 10.4 \\
1 week-1 month & 8 & 6.5 \\
1-6 months & 9 & 10.4 \\
6 months-1 year & 22 & 11.7 \\
1-5 years & 7 & 28.6 \\
5-10 years & 18 & 9.1 \\
$>10$ years & & 23.4 \\
“Getting sober” & 24 & \\
1 time (1st time) & 15 & 31.2 \\
2 times (2nd time) & 16 & 19.5 \\
3-5 times & 7 & 20.8 \\
6-10 times & 15 & 9.1 \\
11 times & & 19.5 \\
Sober due to ultimatum & & \\
0 times & 41 & 53.2 \\
1 time & 14 & 18.2 \\
2 times & 9 & 11.7 \\
3-5 times & 12 & 15.6 \\
6-10 times & 0 & 0.0 \\
\hline & &
\end{tabular}

Since our sample included individuals who used a variety of substances we sought to explore any potential differences in perceptions of ultimatums that may be a consequence of the drug of choice of the participant using a one-way analysis of variance.

Drug of choice was associated with a significant difference in the degree to which an individual believed ultimatums are useful, $\mathrm{F}(3,70)=2.92, \mathrm{p}=0.04$. Post hoc analyses using the least squared difference method revealed that individuals who preferred crack/cocaine $(\mathrm{M}=4.90)$ believed ultimatums to be significantly more useful than did individuals who prefer alcohol $(\mathrm{M}=3.28 ; \mathrm{p}=0.023)$ and polysubstance users $(\mathrm{M}=2.14 ; \mathrm{p}=0.059)$. There was also a non-significant trend for individuals who prefer crack/cocaine to see ultimatums as more helpful than those who prefer heroin/opiates $(\mathrm{M}=3.00 ; \mathrm{p}=0.059)$. While drug of choice was associated with group differences in perceptions of ultimatums, a history of having entered treatment due to an ultimatum was not associated with a significant difference, $\mathrm{t}(75)=0.87, \mathrm{p}=0.387$ ).

Experiences with ultimatums and relapse: We wanted to explore the relationship between receiving ultimatums and sobriety experience. Number of attempts at sobriety was positively associated with number of times an individual got sober due to an ultimatum $(\mathrm{r}=0.51, \mathrm{p}<0.001)$ while the length of current sobriety was not $(r=-18, p=0.114)$. In other 
Current Research in Psychology 1 (1): 29-34, 2010

words, receipt of an ultimatum was not effective at preventing relapse since individuals who regularly sought sobriety after ultimatums were likely to also be those individuals who experienced relapse most often. While more ultimatums would accompany more relapses, the ultimatums were not preventing future relapse. Not surprising, number of attempts at sobriety was negatively associated with the length of current sobriety $(r=-0.28, p=0.013)$. Length of sobriety, getting sober due to an ultimatum and number of attempts at sobriety were all unrelated to perceptions of helpfulness of ultimatums for the individual specifically $(-0.14,0.09$ and 0.03 , respectively) and for people in general $(-0.06,0.00$ and -0.07 , respectively).

Usefulness of and problems with ultimatums: In order to allow participants to provide additional information outside of their responses to closed ended questions, we asked participants to write freely about the benefits and problems with ultimatums. We opted to use emergent coding to analyze these responses in order to allow the themes to reflect the voice of participants. Thus, emergent coding was used to evaluate responses to open-ended questions regarding perceived benefits and problems with ultimatums, as well as the most helpful and unhelpful aspects of personal experiences with ultimatums (Table 2 for list of themes).

Six themes emerged when participants were asked about their perceived benefits of ultimatums. These themes include that there are no benefits and treatment is a matter of individual choice, that ultimatums produce a fear of consequences for using, that ultimatums bring about awareness of the problem, that ultimatums encourage initial treatment, that they are beneficial for the family members and that they cause addict to realize they have support (Table 2). About half of respondents (39) reported a belief that there were no benefits of receiving ultimatums (e.g., "Absolutely NONE. It is a horrible waste of time for both the user and the family and can only hurt both. It can tear everyone apart. The only way to successfully stop drinking is to do it for yourself and as much by yourself as possible"). A number of respondents also reported ultimatums bringing about feared consequences (15), awareness of the problem (14) and initial treatment (13).

When asked about problems with ultimatums, 26 individuals mentioned that there was a lack of commitment from the user (e.g., "The commitment is not there unless the person chooses to accept responsibility for their lives, health and happiness").
Table 2: Perceived benefits of and problems with ultimatums

\begin{tabular}{|c|c|c|}
\hline & $\begin{array}{l}\text { No. of } \\
\text { individuals }\end{array}$ & $\begin{array}{l}\text { Respondents } \\
(\%)\end{array}$ \\
\hline \multicolumn{3}{|l|}{ Benefits of ultimatums } \\
\hline None-individual choice & 39 & 50.6 \\
\hline Fear of consequences & 15 & 19.4 \\
\hline Awareness of problem & 14 & 18.2 \\
\hline Initial treatment & 13 & 16.8 \\
\hline Beneficial for family members & 4 & 5.1 \\
\hline Realization of support & 4 & 5.1 \\
\hline \multicolumn{3}{|l|}{ Problems with ultimatums } \\
\hline No. commitment from user & 26 & 33.7 \\
\hline Resentment & 16 & 20.7 \\
\hline Relapse/rebellion & 15 & 19.4 \\
\hline No problems & 9 & 11.6 \\
\hline Loss of relationships & 8 & 10.3 \\
\hline Cause guilt/shame & 6 & 7.7 \\
\hline \multicolumn{3}{|l|}{ Helpful personal ultimatum* } \\
\hline None helpful & 14 & 18.1 \\
\hline Threat of losing family members & 13 & 16.8 \\
\hline Brought about awareness & 11 & 14.3 \\
\hline Court mandate & 6 & 7.7 \\
\hline Threat of losing job/home & 3 & 3.8 \\
\hline Threat of losing life & 2 & 2.5 \\
\hline \multicolumn{3}{|l|}{ Unhelpful personal ultimatum* } \\
\hline Given in judgment/wrong attitude & 13 & 16.8 \\
\hline Choice taken/not ready & 9 & 11.6 \\
\hline Ultimatums always helpful & 4 & 5.1 \\
\hline No follow through & 3 & 3.8 \\
\hline Forced out of treatment preference & 3 & 3.8 \\
\hline Lying to draw person to intervention & 2 & 2.5 \\
\hline
\end{tabular}

*: Not all respondents reported personal experiences with ultimatums and were not included in table

Other themes include resentment (e.g., "If a person is not ready to get sober an ultimatum can drive a huge wedge into the relationship and the resentment over this can keep a person in their using"), relapse and rebellion, loss of relationships and causing guilt/shame for the addict. In addition, 9 individuals reported no perceived problems with giving/receiving ultimatums.

We opted to ask those participants who had personally experienced an ultimatum to provide details about their own experiences with such an approach to their addiction from family members or friends. Participants that had personally experienced ultimatums reported six themes of helpful ultimatums. These included no aspects of the ultimatum being helpful (14), the threat of losing family members being helpful (e.g., "Get clean or you can't see your daughter.... It motivated me to get clean and stay clean"), ultimatums that brought about personal awareness (11), court mandates (6) and the threat of losing a job, home, or life (due to illness). Finally, individuals that had personally experienced ultimatums reported six themes of unhelpful ultimatums. Thirteen individuals reported the ultimatum given in judgment or with the wrong attitude being unhelpful (e.g., "People being judgmental of me. Withdrawing support from an ill 
person implies that their illness is a moral choice they are making"). Other themes included the individual not being ready when the ultimatum was given (9), no follow through on the threats made by the ultimatum (3), forced out of treatment preference (e.g., "I suppose during a time in rehab being forced to engage in AA when my choice in recovery is SOS") and lying to get individual to the ultimatum (2). Four individuals experienced only helpful ultimatums.

\section{DISCUSSION}

This study sought to explore whether individuals suffering from addiction were more likely to relapse if they had entered treatment due to an ultimatum as opposed to entering treatment by their own choice. We were also interested in individual perceptions of ultimatums in general and based on personal experience. Results showed that individuals who regularly sought treatment due to an ultimatum were likely to relapse after seeking treatment. This suggests that ultimatums are not effective in preventing relapse in individuals. Although ultimatums appear unhelpful in preventing relapse, respondents differed in their perceptions of the helpfulness of ultimatums depending on their drug of choice, with those who reported crack/cocaine as their primary substance of choice perceiving ultimatums as more helpful than individuals who identified alcohol as their primary substance of choice. Despite this finding, emergent coding revealed that a great number of individuals did not perceive ultimatums as beneficial in general and that many of those with personal experiences with ultimatums did not find them helpful.

Limitations: This study contains several important limitations including the use of a restricted sample, reliance upon self-report of respondents and lack of ability to draw inferences regarding causal relationships. The choice to use an on-line sample was made to protect the anonymity of respondents. Since approaching individuals in support groups such as those attending a meeting for Alcoholics Anonymous could be seen as potentially intrusive and would violate individuals' privacy prior to obtaining informed consent, the use of on-line participants was deemed appropriate as an initial step in exploring IWA's perceptions of ultimatums. However, only those who use such support groups would have been invited to participate in the study. As such, the results of this study may not generalize well to all IWAs. In addition, the study required participants to recall specific details regarding their treatment, sobriety, relapses, substance use and experiences with ultimatums. It is not possible to verify the accuracy of participants' responses and studies initiated in treatment facilities where histories are obtained from both IWAs and significant others would increase confidence in these findings. Finally, while we found a link between relapse frequency and history of ultimatums, it is not possible to know whether ultimatums increase risk of relapse or whether people experience more ultimatums because they relapse more frequently. In reality it is likely that the relationship is somewhat bidirectional and other factors such as problematic family environments may contribute to both. Although causal studies to evaluate factors contributing to relapse would be problematic, larger studies that consider alternative causal factors will help further illuminate this relationship.

Implications: These findings have some important implications for the use of ultimatums as seen on the television series Intervention. Although ultimatums are extremely effective for getting individuals to enter treatment, these individuals are likely to relapse. Frequent relapse may incite a greater number of, eventually less effective, ultimatums. Therefore, it is important to assess whether ultimatums should be used to encourage users to seek treatment and if so, the most appropriate times to use them. Such information would allow family members and friends to truly act in the best interest of the user. Additionally, helpfulness of ultimatums may differ depending on the primary drug of choice. Crack/Cocaine users may respond more readily to ultimatums than other IWAs. This suggests that interventions may need to be modified to fit the profile of the substance user rather than assuming ultimatums will be effective in all cases. Lastly, it is important to look at evidence that may contradict the use of ultimatums in general. Familial relationships have been shown to increase capacity for sobriety (Lavee and Altus, 2001). However, many participants report ultimatums being unhelpful, particularly in the fact that commitment to change is not present on the part of the addict and relationships are lost in the process of giving ultimatums.

\section{CONCLUSION}

The findings in this study provide an initial picture regarding the usefulness of ultimatums with IWA's; however, future research will need to address some important limitations of this study. First, particular characteristics of the IWA's and their ultimatum experiences will need to be evaluated to identify specific aspects that made ultimatums unsuccessful. This study provides a small contribution to the research 
regarding drug of choice and its' effect on the perceptions of ultimatums. However, other characteristics such as the IWA's environmental resources, the relationship with members of the social support system, the family's commitment to the intervention process and the intervention specialists' qualifications/training (if a specialist was present) may all be indicators of whether an ultimatum would be beneficial to an IWA. This research may differentiate between successful (with success defined as both obtaining and sustaining sobriety) and unsuccessful ultimatum characteristics which could help to build an understanding of the type of IWA that will most benefit from or be harmed by ultimatum-driven interventions. Additionally, the scope of this research was not able to address other treatment interventions that may work in lieu of ultimatums. If an IWA will not benefit from an ultimatum-driven intervention, are there other options available that may be more beneficial?

It seems necessary that ultimatums should be used in certain circumstances. However, with the perceived unhelpfulness of ultimatums that exists on the part of IWAs, coupled with the likelihood of relapse after ultimatums, research evaluating when ultimatums are necessary is clearly needed. In addition, such research would need to explore when ultimatums can be implemented with the greatest level of efficacy.

\section{ACKNOWLEDGMENT}

Many thanks to the individual members of the multiple on-line addiction support groups whose participation was critical to this study. A limited portion of this manuscript was presented as a poster at the American Psychological Association Convention 2008, Division 42.

\section{REFERENCES}

Barber, J.G. and B.R. Crisp, 1995. The 'pressures to change' approach to working with the partners of heavy drinkers. Addiction, 90: 269-276. PMID: 7703820

Campbell, W.G., 2007. Addiction is treated by all domains of our society. Can. J. Psychiatry, 52: 170-172. http://www.ncbi.nlm.nih.gov/pubmed/17479525

Carroll, K.M., B. Libby, J. Sheehan and N. Hyland, 2001. Motivational interviewing to enhance treatment initiation in substance abusers: An effectiveness study. Am. J. Addict., 10: 335-339. http://www.ncbi.nlm.nih.gov/pubmed/11783748

Copello, A.G., R.D. Velleman and L.J. Templeton, 2005. Family interventions in the treatment of alcohol and drug problems. Drug Alcohol Rev., 24: 369-385. PMID: 16234133
Hall, J.M., 1993. What really worked? A case analysis and discussion of confrontational interventions for substance abuse in marginalized women. Arch. Psychiatry. $\quad$ Nurs., $7: \quad 322-327$. http://www.ncbi.nlm.nih.gov/pubmed/8179355

Laudet, A.B., 2003. Attitudes and Beliefs about 12-step groups among addiction treatment clients and clinicians: Toward identifying obstacles to participation. Substance Use Misuse, 38: 2017-2047. http://cat.inist.fr/?aModele=afficheN\&cpsidt=15318576

Lavee, Y. and D. Altus, 2001. Family relationships as a predictor of post-treatment drug abuse relapse: A follow-up study of drug addicts and their spouses. Contemp. Family Ther. Int. J., 23: 513-530. http://cat.inist.fr/?aModele $=$ afficheN\&cpsidt $=13412676$

Loneck, B., J.A. Garrett and S.M. Banks, 1996. The Johnson Intervention and relapse during outpatient treatment. Am. J. Drug Alcohol Abuse, 22: 363-375. http://www.ncbi.nlm.nih.gov/pubmed/8841685

Marshall, J.P., T.G. Kimball, S.T. Shumway, M.M. Miller and V. Jeffries et al., 2005. Outcomes of a structured family group in an outpatient alcohol/other drug treatment setting. Alcohol. Treat. Q., 23: 39-53. http://direct.bl.uk/bld/PlaceOrder.do?UIN=183038 423\&ETOC $=$ RN\& from=searchengine

Matzger, H., L.A. Kaskutas and C. Weisner, 2005. Reasons for drinking less and their relationship to sustained remission from problem drinking. Addiction, $\quad 100$ : 1637-1646. http://cat.inist.fr/?aModele=afficheN\&cpsidt=17213147

Merriam-Webster, 2003. Merriam-Webster's Collegiate Dictionary. 11th Edn., Merriam-Webster Inc., Springfield, MA., ISBN: 0877798095, pp: 1664.

Meyers, R.J., M. Villanueva and J.E. Smith, 2005. The community reinforcement approach: History and new directions. J. Cognit. Psychother., 19: 247-260. http://www.highbeam.com/doc/1P3-913174411.html

Polcin, D.L. and M. Beattie, 2007. Relationship and institutional pressure to enter treatment: Differences by demographics, problem severity and motivation. J. Stud. Alcohol Drugs, 68: 428-436. http://www.ncbi.nlm.nih.gov/pubmed/17446983

Prochaska, J.O., C.C. DiClemente and J.C. Norcross 1992. In search of how people change, applications to addictive behaviors. Am. Psychol., 47: 11021114. PMID: 1329589

Saatcioglu, O., R. Erim and D. Cakmak, 2006. Role of family in alcohol and substance abuse. Psychiatry Clin. Neurosci., 60: 125-132. PMID: 16594934

Stead, P. and J. Viders, 1979. A 'SHARP' approach to treating alcoholism. Soc. Work, 24: 144-149. http://www.ncbi.nlm.nih.gov/pubmed/10240954 